\title{
Abrasive Flow Machining: An Area Seeking for Improvement
}

\author{
Rajendra Baraiya ${ }^{1}$, Vivek Jain ${ }^{1}$, Dheeraj Gupta ${ }^{1}$ \\ ${ }^{I}$ Department of Mechanical Engineering, Thapar University, Patiala, Punjab, India - 147004
}

\begin{abstract}
Abrasive Flow Machining (AFM) was developed as a method to deburr, polish, and chamfer very hard to reach surfaces like perplexed geometries and edges by flowing abrasive loaded viscoelastic polymer over them. Abrasion occurs wherever the medium passes through the highly restrictive passage. Process input parameters such as extrusion pressure, number of cycles, grit composition and type, tooling and fixture designs have effect on AFM output parameters such as surface finish and material removal. The correct selection of manufacturing conditions is one of the most significant aspects to take into concern in the majority of manufacturing processes and mainly in processes related to Abrasive Flow Machining. The present paper highlighted some of the existing techniques of AFM and its process parametric characterization.
\end{abstract}

Keywords - Abrasive flow machining, Abrasives, Surface Finish

\section{INTRODUCTION}

Abrasive Flow machining (AFM) is a non-traditional machining method that can be used to deburr, chamfer, polish, remove recast layers, and to produce compressive residual stresses. Abrasive Flow Machining (AFM) was first described and patented by McCarty, the president of Extrude Hone Corporation in 1970 [1]. The most time consuming and labour demanding section of the manufacturing process in today's industry is the final finishing of complex and precision works. This consumes as much as $5-15 \%$ spending of the overall manufacturing Process. Because of its capability to machine interior and curved surfaces, it is considered as a suitable surface finishing process to finish internal complex geometry of workpiece. The process can deburr holes as small as $0.2 \mathrm{~mm}$ and radius edges from 0.025 to $1.5 \mathrm{~mm}$. Tolerances can be held to $\pm 5 \mu \mathrm{m}$. AFM is becoming popular due to its ability to give expected, constant and repeatable results [3].

\subsection{Principles of AFM}

In the AFM process, a semi- solid media is utilized which comprises of a carrier in the form of a polymer base containing abrasive powders in a desired proportion, which is extruded under the given pressure across the surface, which is to be machined. The media acts as a flexible tool whenever it is subjected to some restrictions due to the uneven surface. The special deformable facility of media is responsible for its movement through any shape of the passage. Restricted media flow passages are obligatory at the surfaces to be processed by AFM, wherein the media deports remotely like flexible grinding stone, abrades the material, and provides a good surface finish over the surface. Generally, a fixture is required to offer restriction or to direct and focus the media to desired locations in the work piece.
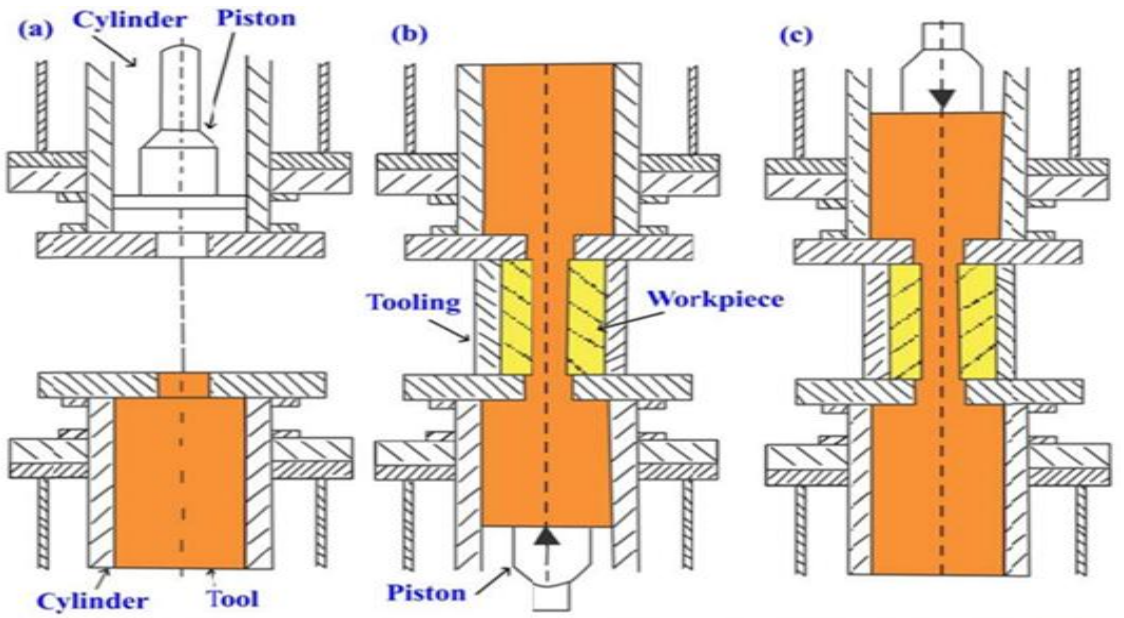

Fig.1 Schematic diagram for Principle of AFM 


\subsection{Classification of AFM}

AFM process can be principally classified in two classes:

\subsubsection{One-way AFM}

One way AFM [7] process apparatus is provided with a hydraulically triggered reciprocating piston and an extrusion media cavity revised to receive and extrude medium uni-directionally across the internal surfaces of a workpiece having internal passageways formed therein. Fixture directs the flow of the medium from the extrusion medium chamber into the internal passages of the workpiece, while a medium collector collects the medium as it extrudes out from the internal channels. The extrusion medium chamber is provided with an access port to intermittently receive medium from the collector into extrusion medium chamber. The hydraulically actuated piston occasionally withdraws from its extruding position to open the extrusion medium chamber access port to collect the medium in the extrusion medium chamber. When the extrusion medium chamber is charged with the working medium, the operation is resumed.

\subsubsection{Two-way AFM}

Two way AFM [6] machine has two hydraulic cylinders and two media cylinders. The media is extruded, hydraulically or mechanically, from the filled chamber to the empty chamber via the controlled passage through or past the workpiece surface to be abraded. Typically, the medium is extruded to and fro between the chambers for the desired fixed number of cycles. Counter bores, recessed areas and even blind cavities can be finished by using restrictors or mandrels to direct the medium flow along the surfaces to be finished.

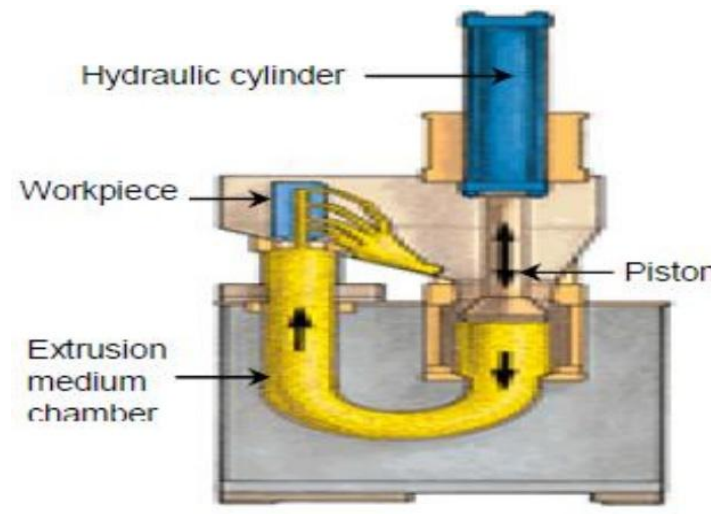

Fig.2: One-Way AFM

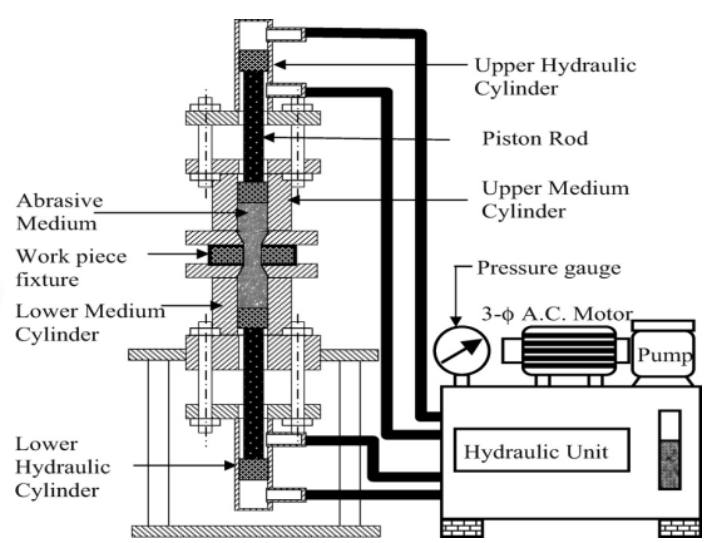

Fig.3: Two-way AFM

II. AFM Process Parameters

The AFM process parameters are classified as given below [31],

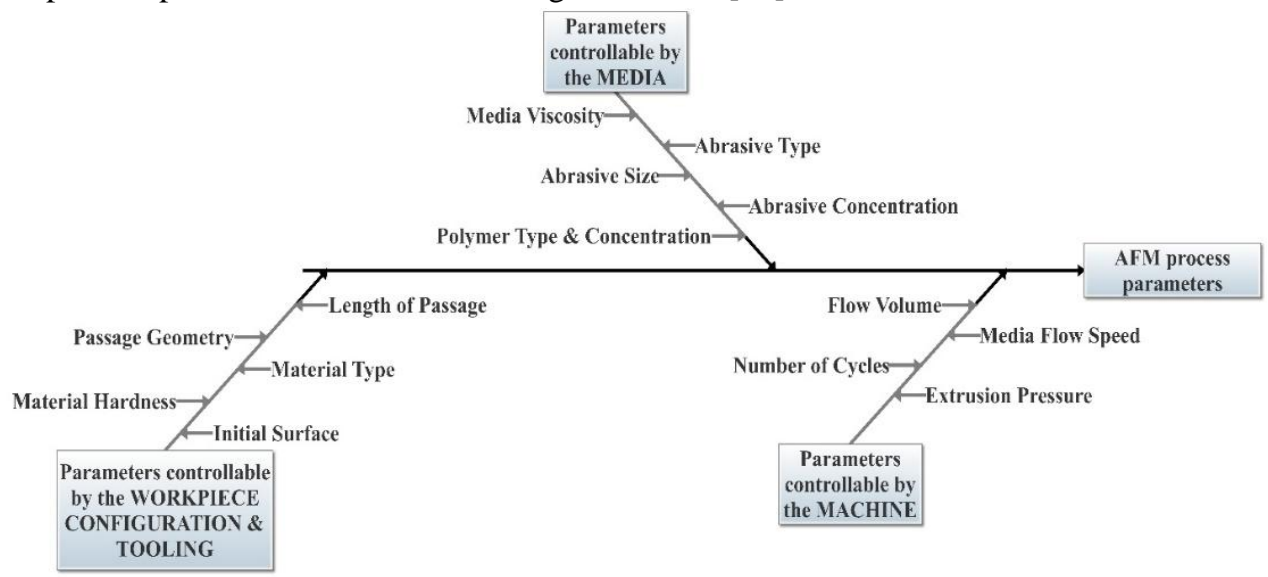

Fig.4: Representation of AFM process parameters in Fish-Bone Diagram 


\subsection{Process Parameters and their Effect on Output Responses}

Experimental investigations have been carried out by various investigators to investigate the effects of process parameters like extrusion pressure, number of cycles, viscosity, abrasive concentration and grain size on the output responses namely, surface finish and material removal during AFM. Rhoades studied the basic principle of AFM and reported that the depth of cut primarily depends upon abrasive grain size, relative hardness and sharpness and extrusion pressure [2-4].

The axial forces in AFM are highly subjected to the behaviour of the AFM medium. The flow pattern of the AFM medium depends on the machining parameters, the characteristics of the applied medium, as well as the shape of the work piece and the tooling [4]. Loveless discussed the effects of AFM on surfaces produced by turning, milling, grinding, and wire electrical-discharge machining. They stated that the best enhancement in the surface quality was obtained on the WEDMed surfaces. The results show that the type of machining process affected both metal removal and surface finish results and medium viscosity significantly affected only surface improvement, while extrusion pressure did not have a significant effect in this article [10]. Jain et al. has built an estimation of material removal and surface roughness obtain during AFM, and found that increasing an piston speed, fraction concentration of grain, extrusion pressure would increase material removal and decrease the surface roughness value, while further than a certain value of velocity and pressure, it would worsen due to increase in depth of indentation, and the minimum surface value is equal to the critical surface roughness. The authors use neural network for modelling and optimum selection of input parameters of AFM process [11, 12].

Jain and Adsul studied the AFM process parameters, such as four varieties of mesh sizes and concentrations, the number of process cycles, different media flow speeds on aluminum and brass, simulating soft and hard materials, respectively. They concluded that leading parameters are the number of process cycles, mesh size and abrasive concentration, and the effect of flow rate is less in comparison to the other AFM process parameters [13]. Jain et al. uses the stochastic simulation to measure the active grain density in AFM. Active grain density increases with an increase in abrasive concentration \& active grain density is higher for a smaller grain diameter for a specific concentration [17].

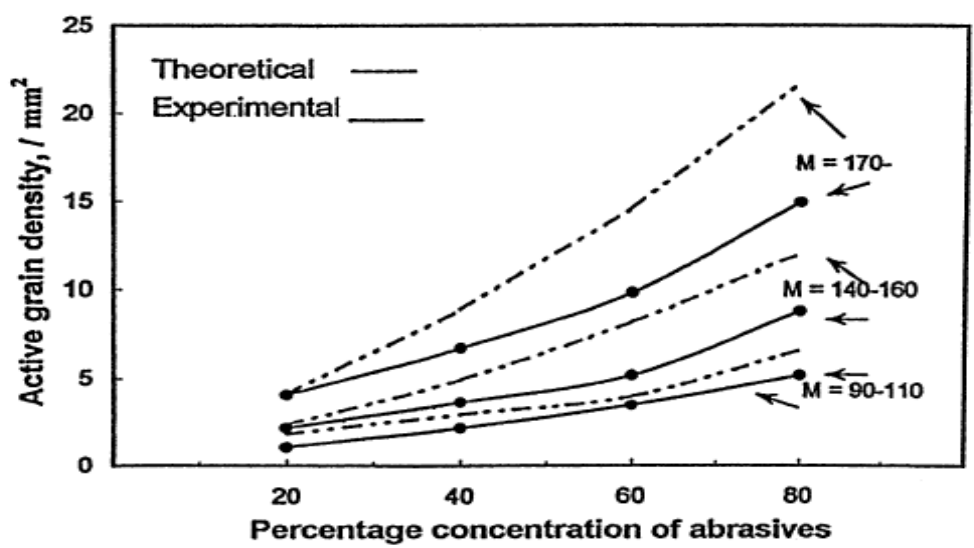

Fig.5 Variation of active grain density with percentage concentration and mesh size [17]

Morochkin suggested three different regimes of grain-workpiece interfaces namely, chip regime, plastic regime and elastic regime [8]. Gorana et al. examined the axial and radial forces during AFM using a self-designed two-component disc dynamometer. It was concluded that extrusion pressure, abrasive concentration and grain size affect the cutting forces, active grain density and finally reduction in surface roughness. They found that the applied extrusion pressure had the strongest effect on the measured axial force. The active grain density and the concentration of abrasive grains showed only a minor effect in their experiments. Author concluded that reduction in surface roughness is approximately linearly proportional to force ratio $(\mathrm{Fr} / \mathrm{Fa})$. Scanning electron microscopy shows that rubbing and ploughing are the possible mechanisms of material deformation [18]. 


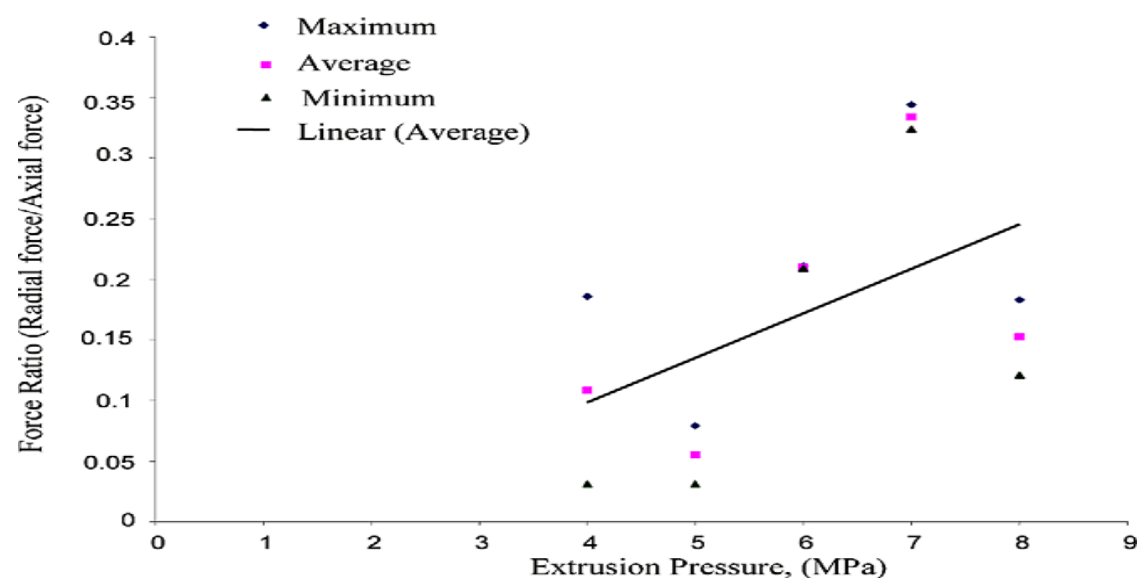

Fig.6 Variation of force ratio with extrusion pressure [18]

In another paper, Gorana et al. studied the modes of material distortion under realistic conditions of grain-workpiece interface. The AFM experimental results showed that axial force, radial force, active grain density and grain depth of indentation, all have a significant effect on the scale of material deformation. Results suggested that care should be taken when evaluating and interpreting the force on a single grain followed by grain depth of indentation which is used in estimate of mode of material deformation. The two established grain-workpiece interaction parameters, viz., the minimum depth of indentation and minimum load required for chip formation, were found to correlate well with the mode of material deformation. The theoretical and investigational results showed that the rubbing mode of material deformation dominates in the study; however, some evidences of ploughing during AFM are also present. They conducted Scratching experiments to simulate and acquire the knowledge about the action of abrasive grains during the AFM process [19].
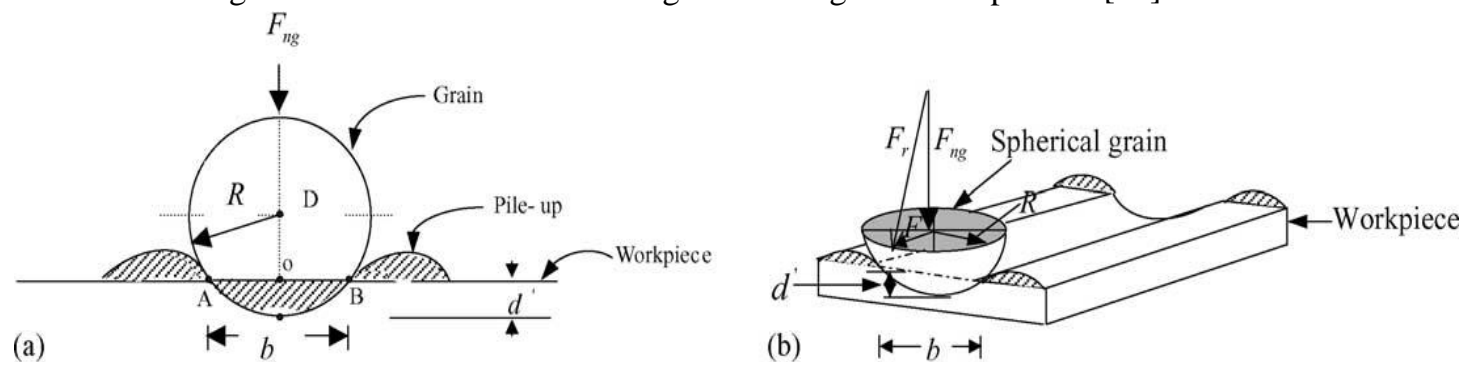

Fig.7 :(a) Indentation of a grain into the workpiece with side pile-up and

(b) Hemisphere normal to the direction of motion [19]

Fang et al. studied abrasive particle movement pattern as an significant factor in approximating the wear rate of materials, especially, as it is closely related to the burring, buffing and polishing efficiency of the abrasive flow machining (AFM) process. There are generally two kinds of particle movement patterns in the AFM process, i.e. sliding-rubbing and rolling [21].

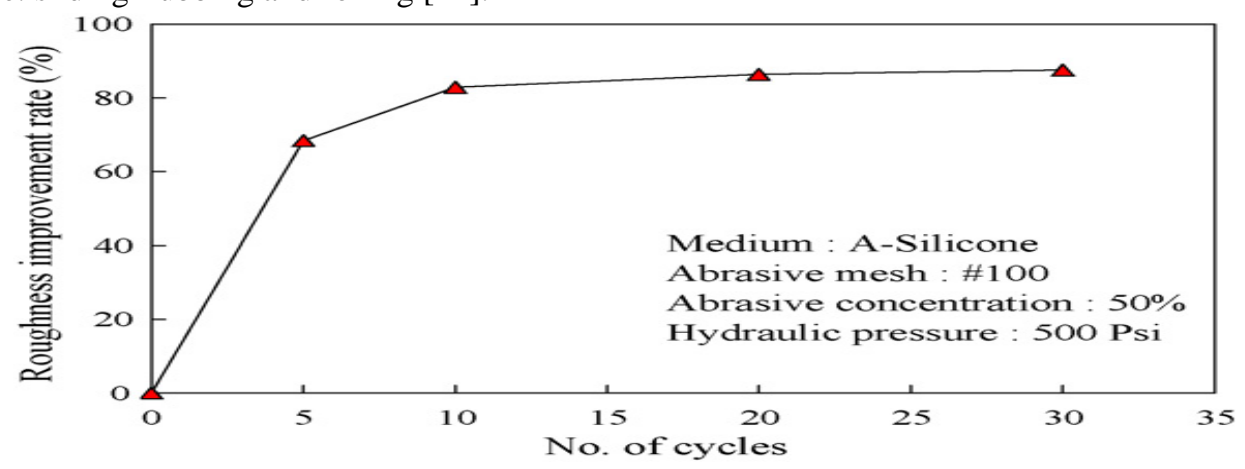

Fig.8: Effect of no. of cycles on the roughness improvement rate [22] 
Wang et al. studied that effect of abrasive gel on the surface roughness \& efficiency of media in material removal. They proposed that Vinyl-silicone polymer (or silicone rubber) as a best material for media because it is cost effective. They also concluded that $80 \%$ surface improvement occur within the $5^{\text {th }}$ number of cycle. Small abrasive sizes can produce smoother surfaces than large sizes [22].

Song et al. discussed the temperature dependence and its effect on surface roughness. Experimental results show that the media at high temperature results in less improvement in surface roughness. Author concluded that media can have good machining performance in the first few cycles and then media temperature rise rapidly. Finally they conclude that the best workable temperature should be below $25{ }^{\circ} \mathrm{C}$ during the AFM [23].

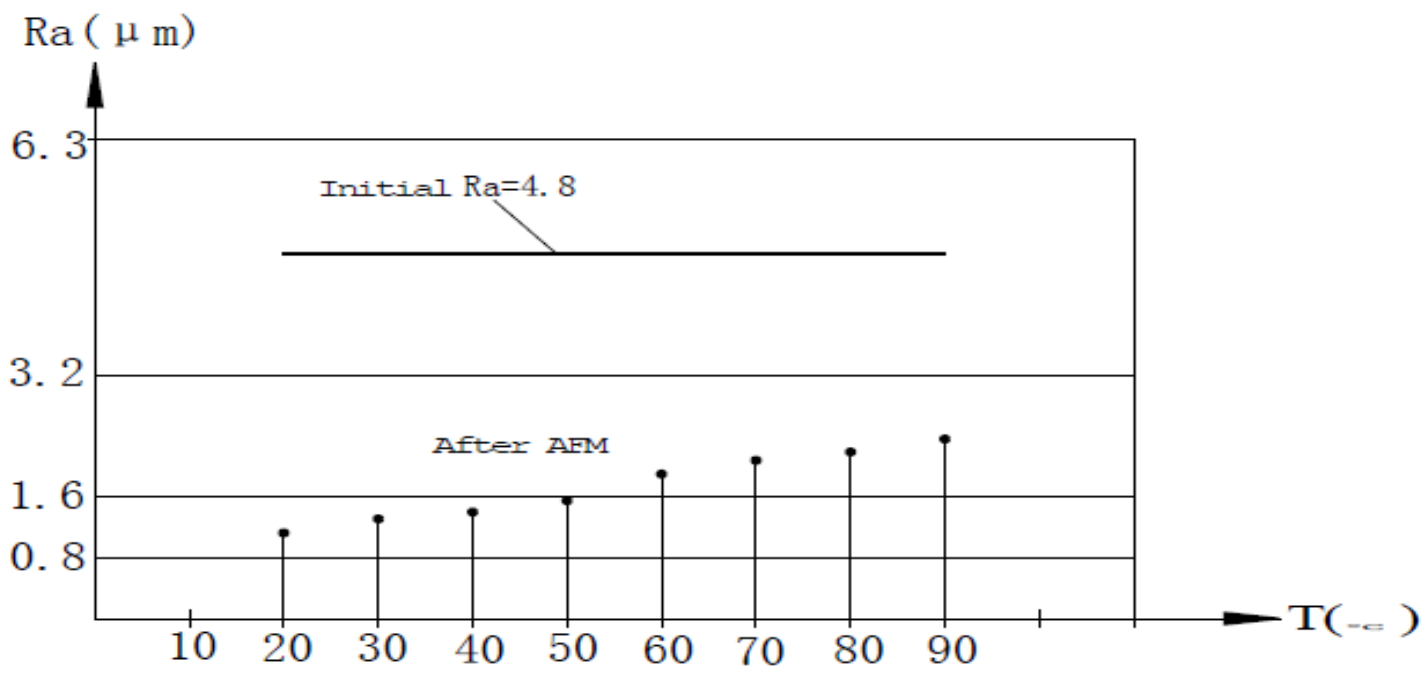

Fig.9: Effect of temperature on surface roughness [23]

Fang et al. studied on the work efficiency of AFM based on the media temperature. They states that media viscosity decreases continuously with increasing temperature. Media temperature increases with increasing cycles, which means media viscosity decreases with cycles increasing. Work efficiency directly proportional to the media viscosity, with decrease in media viscosity rolling tendency of abrasive particle is increase, which cause decrease in work efficiency [24].

Kara Kamal K. et. al. developed substitute media for AFM process from different viscoelastic carriers (natural rubber and butyl rubber), $\mathrm{SiC}$ abrasive and naphthenic oil. The performance of media was evaluated by an AFM setup. The butyl rubber, silicon carbide and naphthenic oil mixed media showed good performance compared to natural rubber based media. As the abrasive loading increases, the improvement in surface roughness increases. But at the high percentage (above 78\%) of abrasive loading, the flow becomes difficult as well as carrier acts as inefficient binder for abrasives. Media contains abrasives of mesh size 220 was good compared to the 800 and 1200 mesh sized abrasive in AFM process [25].

M. Ravi Sankar et al. initially grounded the $\mathrm{Al}$ alloy and two types of $\mathrm{Al}$ alloy/SiC MMCs (i.e., 10\% and $15 \%$ volume fractions) and then fine finished using Abrasive flow finishing process. New Styrene Butadiene rubber (SBR) medium was developed and used in the AFF process during finishing of three types of workpiece materials. The following conclusions were made, as the extrusion pressure increases surface roughness increases upto $6 \mathrm{MPa}$ and then decreases slightly on further increment. Surface roughness increases gradually as weight percentage of oil increases upto $10 \%$ and then starts decreasing. As hardness of workpiece increases, the number of cycles to achieve better Surface roughness also increases [26].

Williams et al. found that the metal removal rate of middle hole was $30 \%$ more than that in side holes, when he performed experiments of extrusion abrasive flow for producing the parts of four side holes and a centre hole [9].

Siddiqui et al. considers vent for media outflow in work-piece surface significantly affects performance measures, material removal (MR) and surface roughness (Ra value) in abrasive flow machining. Work-piece 
surfaces having single vent for media outflow by abrasive flow machining produced better results in comparison with work-piece surfaces having multiple vents. Work-piece surfaces having single vent/passage for media outflow have higher material removal and more enhancement in surface roughness and the performance measures decrease with increase in the number of vents for media outflow. The change in surface roughness, $\triangle \mathrm{Ra}$ increases with the increase in length of the work-piece and decreases with the increase in cross section of the work-piece [30]. It is also observed that the pressure decreases with increase in length of media flow travel. In AFM, it has been reported that enhancement in performance could be achieved by improved fixturing [27].

The machining parameters, such as concentration, mesh number and number of cycles were changed to determine their effects on the machining characteristics material removal (MR) and enhancement in surface finish in PEC Chandigarh [29]. Brar et al. concluded that smaller size abrasive gives better surface finish and can reach into complex and narrow passages, while larger one cut faster. The reason for a decrease in material removal is that with an increase in mesh size (or decrease in grain size in $\mathrm{mm}$ ) the depth of penetration as well as width of penetration, decreases. Authors performed parameter study on one-way AFM regarding the effect of the piston pressure on the surface finish. Based on previous findings, two machining strategies with a combination of roughing and finishing are compared to the machining with high and low pressure $[32,33]$

\section{RECENT DEVELOPMENTS IN AFM}

Though there are many advantages of AFM process, it has a few disadvantages also, such as low productivity. The time to achieve the required surface finish is longer in AFM process as compared to other finishing processes. Researchers have tried to overcome this difficulty by using hybrid approach and have reported improvement in process efficiency of AFM.

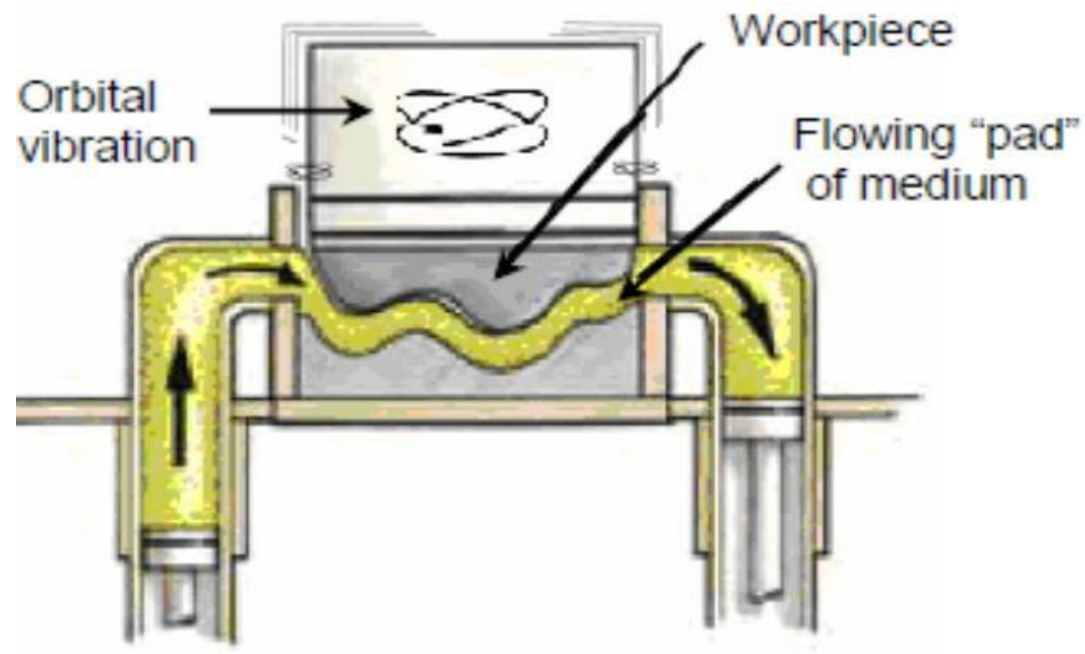

Fig.10: Orbital AFM

Orbital AFM [5] combines abrasive flow machining and orbital grinding. An additional mechanical motion is given to the medium to enable it polish three dimensional forms that are not possible with conventional AFM. The motion is typically a planetary oscillation that creates relative displacement between tooling and the work piece. The oscillations can be in the vertical or horizontal or combination of both the planes, yielding an elliptical or gyratory polishing action. Orbital polishing is made used in polishing the edges and surfaces in complex shapes and cavities such as bottle molds, coining dies and aluminium wheels along with high precision and accuracy.

Singh and Shan applied magnetic field around the workpiece in AFM and observed that magnetic field significantly affect the material removal and change in surface roughness [15]. Then Jha and Jain also apply magnetic field to flowing media in AFM process for further study. Media for MRAFF process contains Abrasives and carrier medium with Carbonyl Iron Particles. Because of effect of magnetic field on the CIPs, the modification of the distribution pattern of abrasive particles occur, hence more number of abrasive particles 
could take part in the abrasion process. They claimed that $20-30 \%$ enhancement in the material removal rate [16]

(a)

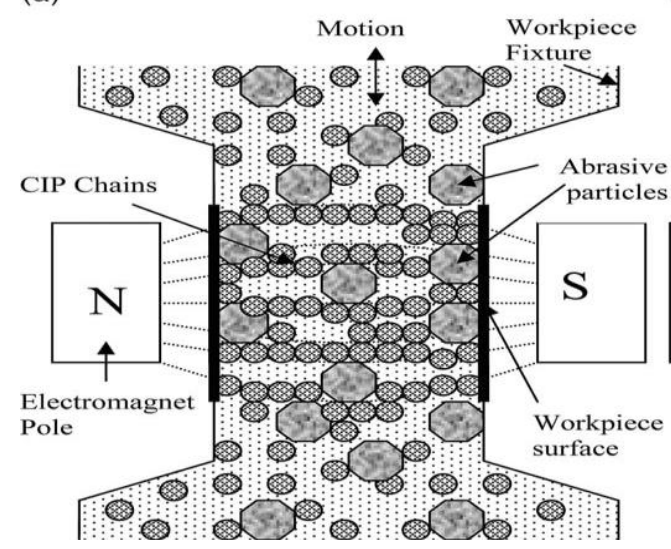

(b)

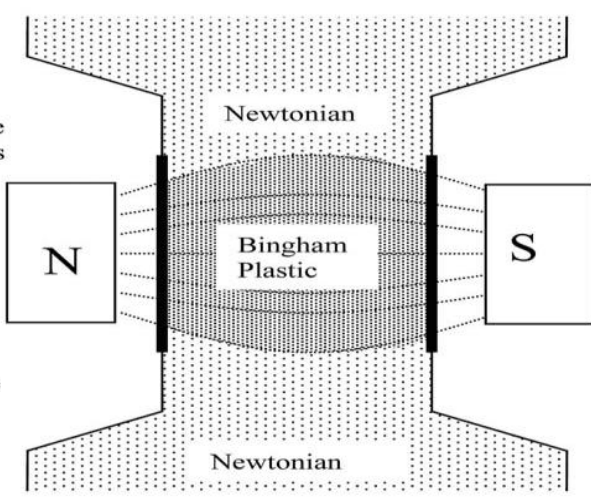

Fig.11: Mechanism of Magnetorheological abrasive flow finishing process [16]

(a)

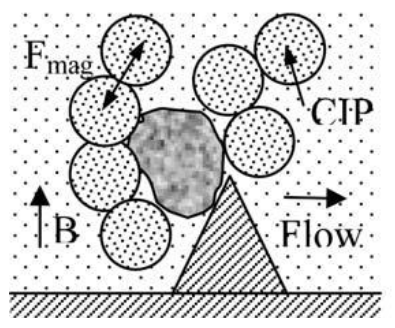

(b)

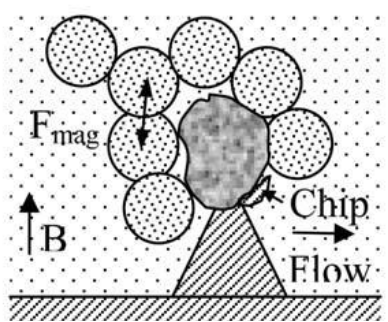

(c)

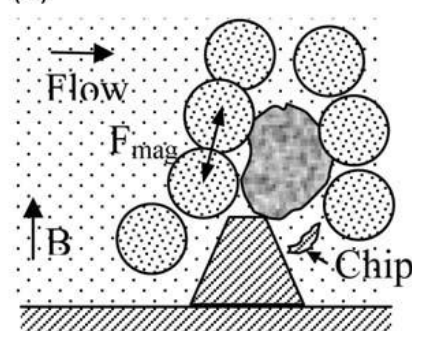

Fig.12: Finishing action on a single profile in presence of external magnetic field [16]

Walia et al. recently explored centrifugal force assisted abrasive flow machining (CFAAFM) process as a hybrid machining process with the aim towards the performance improvement of AFM process by applying centrifugal force on the abrasive laden media with a rotating centrifugal force generating (CFG) rod introduced in the work piece passage [20].

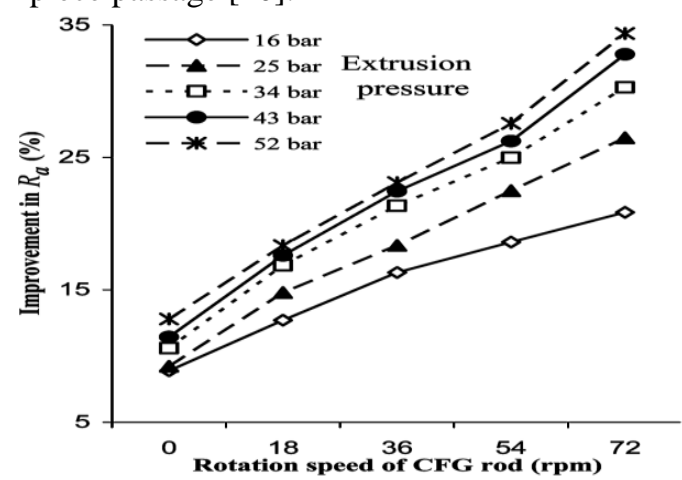

(a)

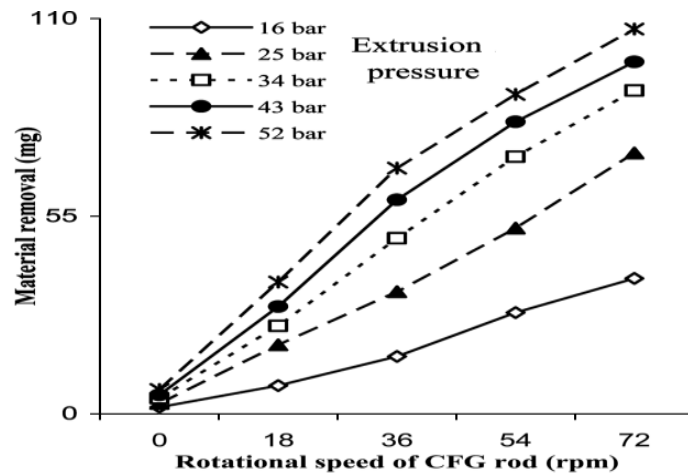

(b)

Fig.13: Effect of CFG rod rotational speed and extrusion pressure on

(a) Improvement in surface roughness (\%) and (b) material removal (mg) [20]

Ravi Sankar et al. developed a new set-up to rotate the workpiece so that the probability of active abrasive particle rotation in the workpiece finishing region is high which improves both surface finishing rate and material removal rate. This study establishes that R-AFF can produce $44 \%$ better $\Delta R a$ and $81.8 \%$ more MR compared to the AFF process [14]. Author tried to improve finishing rate, material removal and surface texture by placing drill bit in the medium flow path called Drill Bit Guided Abrasive Flow Finishing (DBG-AFF). The 
inner part of medium slug flows along the helical flute which creates random motion among the abrasives in inner region of the medium. This causes reshuffling of abrasive particles at outer region. Hence, comparatively more number of new and fresh abrasive grains interact with the workpiece surface. From the experimental results, it is concluded that the abrasive traverse path is longer than the AFM abrasive traverse path in each cycle. It results in higher finishing rate in DBG-AFF as compared to AFM. Material removal is found to decrease with decrease in drill bit diameter [28].

Kendra et al. suggest that the problem of nonuniform polished surface and nonuniform material removal can be solved by using a movable/rotatable mandrel. The innovative upgrade of AFM with movable/rotatable mandrel (AFMmm) shows high potential in improvement of their productivity [34].

Venkatesh et al. works on Ultrasonic Assisted Abrasive Flow Finishing to improve the surface roughness of bevel gear. In UAAFF, abrasive polymer mixture is pumped down the centre of the ultrasonically energized tool. Combination of flow and vibration results in the effective abrasion of the workpiece surface. They takes Velocity, pressure and temperature as an input parameter to measure the surface finish and material removal of the bevel gear in AFM. They concluded that ultrasonic assisted AFM can increase velocity of abrasive, increase active grains hence improve in surface quality and MR rate. UAAFF has capacity to produce micro/nano level finish on closed cavity surfaces without causing much deterioration to its profile or dimensional accuracy. With this process blind hole can be also polished [35]

\section{CONCLUSION}

For improved surface integrity, smoothness and performance, non-stop progresses are taking place for altering the existing AFM process technology and AFM machine structure. To overcome some of the draw backs such as low finishing rate and incapability to correct the form geometry, investigators have proposed various types of AFM machines abbreviated as Orbital AFM, MRAFM, UAAFM, AFMmm, R-AFM, Spiral polishing etc. There is a big need of a process which improves the surface finish externally as well as internally simultaneously. Abrasive flow machining is a potential candidate for this direction.

\section{REFERENCES}

[1] R.W. McCarty, Method of honing by extruding, United States Patent US3521412 (A); 1970.

[2] L.J. Rhoades, "Abrasive flow machining and its use", Proc. Non-traditional Machining Conf., Cincinnati, OH, 1985, 111-120.

[3] L.J. Rhoades, "Abrasive flow machining", Manuf. Eng., 1988, 75-78.

[4] L.J. Rhoades, "Abrasive flow machining: a case study", J. Mater. Process. Technol., 28, 1991,.107-116

[5] Rhoades L.J., Orbital and or reciprocal machining with a viscous plastic medium, International patent no: WO 90/05044, 1990.

[6] L.J. Rhoades, T.A. Kohut, Reversible Unidirectional AFM, US patent number 5,070,652, 1991

[7] L.J. Rhoades, T.A. Kohut, N.P. Nokovich, D.W. Yanda, Unidirectional abrasive flow machining, US patent number 5,367,833, 1994

[8] V.N. Marochkin, The limiting plastic state in indenting and compressing a truncated cone, Friction Wear Mach., 13 ASME, 1959.

[9] R.E. Williams, K.P. Rajurkar, "Metal removal and surface finish characteristics in abrasive flow machining, Mechanics of Deburring and Surface Finishing Processes", New york, ASME, 1989, 93-106.

[10] R. Loveless, R.E. Williams, K.P. Rajurkar, "A study of the effects of abrasive-flow finishing on various machined surfaces", J of Mater Process Technol. 47, 1994,133-151

[11] R.K. Jain, V.K. Jain and P. M. Dixit, "Modeling of material removal and surface roughness in abrasive flow machining process", International Journal of Machine Tools and Manufacture, 39(12), 1999, 1903-1923

[12] R.K. Jain and V.K. Jain, "Optimum selection of machining conditions in abrasive flow machining using neural network", Journal of Materials Processing Technology, 108, 2000, 62-67

[13] V.K. Jain and S.G. Adsul, "Experimental investigations into Abrasive Flow Machining", International Journal of Machine Tool and Manufacturing, 40(7), 2000, 1003-1021

[14] M. Ravi Sankar, V.K. Jain and J. Ramkumar, "Rotational abrasive flow finishing (RAFF) process and its effects on finished surface topography", International Journal of Machine Tools \& Manufacture, 50, 2000, 637-650.

[15] S. Singh, H.S. Shan, "Development of magneto abrasive flow machining process", International Journal of Machine Tool \& Manufacture, 42, 2002, 953-959

[16] S. Jha and V.K. Jain, "Design and development of the magnetorheological abrasive flow finishing (MRAFF) process", International Journal of Machine Tools \& Manufacture, 44, 2004, 1019-1029.

[17] R.K. Jain, V.K. Jain, "Stochastic simulation of active grain density in abrasive flow machining", Journal of Materials Processing Technology, 152, 2004, 17-22.

[18] V.K. Gorana, V.K. Jain and G.K. Lal, "Experimental investigation into cutting forces and active grain density during abrasive flow machining”, International Journal of Machine Tools \& Manufacture, 44, 2004, 201-211. 
[19] V.K. Gorana, V.K. Jain and G.K. Lal, Forces prediction during material deformation in abrasive flow machining, Wear 260, 2006, $128-139$

[20] R.S. Walia, H.S. Shan, P. Kumar, "Abrasive flow machining with additional centrifugal force applied to the media", Machining Science and Technology, 10(3), 2006, 341-354.

[21] L. Fang, K. Sun, Q. Cen, "Particle movement patterns and their prediction in abrasive flow machining", Tribotest, 13(4), 2007, 195206.

[22] A.C. Wang, S.H. Weng, "Developing the polymer abrasive gels in AFM process", Journal of Materials Processing Technology, 192193,2007, p.486-490

[23] G. Z. Song, Y. Z. Li, G. Ya, "Temperature Dependence and Effect on Surface Roughness in Abrasive Flow Machining”, Advanced Materials Research, 53-54, 2008, 375-380.

[24] L. Fang, J. Zhao, K. Sun, D. Zheng, D. Ma, "Temperature as sensitive monitor for efficiency of work in abrasive flow machining", Wear 266, 2009, 678-687.

[25] Kamal K. Kar, N.L. Ravikumar, Piyushkumar B. Tailor, J. Ramkumar and D. Sathiyamoorthy, "Performance evaluation and rheological characterization of newly developed butyl rubber based media for abrasive flow machining process", Journal of materials processing technology, 209 (4), 2009, 2212-2221.

[26] M. Ravi Sankar, J. Ramkumar and V.K. Jain, "Experimental investigation and mechanism of material removal in nano finishing of MMCs using abrasive flow finishing (AFF) process", Wear 266, 2009, 688-698.

[27] R.S. Walia, H.S. Shan and P. Kumar, "Enhancing AFM process productivity through improved fixturing", Int J Advd Mfg Technol, 44, 2009, 700-709.

[28] M. Ravi Sankar, S. Mondal, J. Ramkumar and V.K. Jain, "Experimental Investigations and Modelling of Drill Bit Guided Abrasive Flow Finishing (DBG-AFF) Process", International Journal of Advanced Manufacturing Technology, 42, 2009, 678-688.

[29] Mandeep Singh, "Design and Development of Abrasive Flow Machining Setup, MTech diss., PEC Univ. of Tech., Chandigarh (2010)

[30] S.S. Siddiqui, M Hameedullah, "Abrasive flow machining performance measures on work piece surfaces having different vent/passage consideration for media-outflow", International Journal of Computer Communication and Information System (IJCCIS) -2(1), 2010.

[31] M. Ravi Sankar, V. K. Jain, J. Ramkumar, Abrasive flow machining (AFM): An Overview (2011)

[32] B.S. Brar, R.S. Walia, V.P. Singh, M. Singh, "Development of a robust abrasive flow machining process setup", Proc. Conf. on Advancements and Futuristic Trends in Mechanical and Materials Engineering, India, 2011.

[33] D. Bähre, H. Brünnet and M. Swat, "Investigation of one-way abrasive flow machining and in-process measurement of axial forces", Procedia CIRP, 1, 2012, $436-441$.

[34] J. Kenda, F. Pusavec and J. Kopac, Arrangements and methods for abrasive flow machining, Patent No - WO 2014184067 A1, 2014.

[35] G. Venkatesh, A.K. Sharma and P. Kumar, "On ultrasonic assisted abrasive flow finishing of bevel gears", International Journal of Machine Tools \& Manufacture, 89, 2015, 29-38. 